\title{
Construction d'un marché alternatif de viande bovine fermière
}

Building of an alternative market of farmer's beef meat

\section{Pierre Stassart}

\section{(2) OpenEdition}

\section{Journals}

\section{Édition électronique}

URL : http://journals.openedition.org/economierurale/3166

DOI : 10.4000/economierurale.3166

ISSN : 2105-2581

\section{Éditeur}

Société Française d'Économie Rurale (SFER)

\section{Édition imprimée}

Date de publication : 5 juillet 2005

Pagination : 28-43

ISSN : 0013-0559

\section{Référence électronique}

Pierre Stassart, «Construction d'un marché alternatif de viande bovine fermière », Économie rurale [En ligne], 286-287 | Mars-juin 2005, mis en ligne le 05 mars 2007, consulté le 02 mai 2019. URL : http:// journals.openedition.org/economierurale/3166 ; DOI : 10.4000/economierurale.3166

Ce document a été généré automatiquement le 2 mai 2019.

(c) Tous droits réservés 


\title{
Construction d'un marché alternatif de viande bovine fermière
}

\author{
Building of an alternative market of farmer's beef meat
}

\author{
Pierre Stassart
}

\section{NOTE DE L'AUTEUR}

L'auteur remercie Marc Mormont pour les échanges et ses relectures très stimulantes.

1 Le point de vue adopté dans cet article est celui de la qualité comme résultat de l'interaction entre acteurs plutôt que comme résultat d'une définition qui viendrait, de par sa nature, s'imposer. Sans le démontrer, nous affirmons qu'il n'existe pas a priori quelques acteurs centraux capables de définir et d'imposer une définition monolithique de la qualité. Au contraire, la pluralité des logiques d'action qui prennent en compte l'interaction entre acteurs et objets répond à différentes formes de coordination qui se traduiront alors par différentes définitions de la qualité. Les travaux qui se prêtent le mieux à l'analyse de ces situations mixtes sont ceux qui cherchent à rendre compte, de façon endogène, de la constitution du collectif. C'est le propos que tiennent notamment le courant de l'économie des conventions et celui de la sociologie de l'innovation et des réseaux.

2 Prenant acte de la rationalité limitée des acteurs et de la nécessité de repères partagés, l'économie des conventions montre comment la construction d'accords entre personnes passe par la justification de leur action en référence à des principes généraux. Ces repères, points d'appui propres à accorder des personnes et non lois naturelles, ont ainsi un caractère conventionnel. Ce courant a contribué à mettre en lumière la diversité des processus de construction collective de la qualité, à travers l'établissement de "Conventions de Qualité » (Eymard-Duvernay, 1989 ; Letablier et Delfosse, 1995). Mais cette approche, centrée sur l'analyse des dynamiques des marchés, examine les conditions du maintien par les entreprises d'une certaine cohérence face à la pluralité des logiques d'action représentée par ces conventions de qualité, sans s'intéresser 
directement à leur émergence et aux dynamiques d'innovation qui les sous-tendent. Or, ces dynamiques de qualification sont aussi constitutives des collectifs qui les construisent. Les saisir dans leur dimension provisoire et inachevée impose de nous intéresser à tout ce travail à la fois endogène et exogène qui permet de révéler, plutôt que d'effacer, la construction interactive et relationnelle de la qualité au sein même des filières.

3 Il nous faut donc mobiliser un cadre qui permette d'analyser la construction des intersections nodales de la nature (agricole), des procédés de fabrication, de la qualité alimentaire, du risque et de la consommation, en centrant cette analyse sur les acteurs et les intermédiaires humains et non-humains qui peuplent ces intersections et dont l'agencement collectif est le résultat ou l'effet de ces réseaux agroalimentaires. Ces associations hétérogènes ou hybrides collectifs rompent avec la conception purifiée du naturel et $\mathrm{du}$ social et décrivent l'inévitable socio-matérialité des processus de qualification que l'on considère dès lors comme des opérations de traduction (Callon, 1989). Ce tournant réflexif (Goodman, 2004) a révélé la richesse conceptuelle et le potentiel de la théorie de « l'acteur-réseau » dans les études agroalimentaires. Il a permis d'ouvrir un nouveau champ d'études empiriques portant plus particulièrement sur les réseaux agroalimentaires dits « alternatifs » (Wathmore et Thorne, 1997, Lockie et Kitto, 2000). Mais le potentiel de cette approche reste encore à explorer notamment parce qu'elle a souffert d'un biais agraire qui privilégiait le point de vue issu de la production, au détriment de la question centrale des consommateurs dans la construction de ces alternatives agroalimentaires (Goodman et Dupuis, 2002). Néanmoins, en suivant la terminologie utilisée, il nous apparaît qu'un autre «missing guest » est demeuré absent, contribuant ainsi à entretenir la discontinuité ontologique que l'étude des réseaux sociotechniques s'attache à résorber : l'intermédiaire.

4 Notre question empirique est celle de la rencontre entre deux collectifs, un collectif de producteurs et d'intermédiaires et de ses conséquences sur la qualification du produit. Ce que nous tentons de cerner, c'est la manière dont s'effectue l'allongement d'un réseau passant d'une situation de face-à-face, producteurs-consommateurs (marché fermier) à une situation dans laquelle des intermédiaires transformateurs interviennent (des bouchers). L'insertion des intermédiaires est-elle une simple importation de compétences et de savoir-faire ou va-t-elle de par la force des réseaux qui la constituent imposer une traduction à portée plus générale dont l'effet ultime serait de dissoudre les spécificités du face-à-face dans des repères plus généraux? Le produit irait-il ainsi vers une standardisation croissante? Au contraire, existerait-t-il une troisième voie qui permettrait simultanément au produit de circuler grâce au travail de l'intermédiaire tout en conservant les spécificités de sa traduction initiale? En terme de définition de la qualité, c'est donc la question de la stabilisation de la spécification du produit liée à la rencontre entre deux réseaux qui nous importe (Murdoch et al., 2000). Ceci pose alors la question de l'hybridation d'un réseau agroalimentaire alternatif lorsqu'il rencontre un collectif «conventionnel », hybridation qui résulterait du rapport entre la capacité de l'un à imposer la généralité de ses références d'une part et la capacité de résistance du collectif innovant.

5 Le cas que nous allons suivre est celui d'une coopérative paysanne belge qui réunit à la fin des années 1970 une quinzaine de paysans pour commercialiser sur les marchés une gamme de produits fermiers "bruts » : poulet, beurre et yaourt, légumes et pommes de terre. Le poulet fermier est alors leur porte-parole (Callon, 1991) parce qu'il traduit la 
qualité fermière qui porte simultanément sur le produit et la relation aux consommateurs. Coprosain, cette coopérative, est attachée comme son nom l'indique au caractère "sain» du produit: poulet à croissance lente, parcours libre, sans antibiotiques, et à la relation directe avec le consommateur : vente directe, groupements d'achat et marchés. Partant de cette traduction initiale de la qualité que nous appellerons traduction «fermière ", nous allons suivre dans la première partie comment l'acquisition d'une boucherie, par la coopérative fermière, entraîne une controverse dont le résultat est d'imposer une seconde traduction, la traduction «bouchère ». Dans la seconde partie, nous décrivons par un retour en arrière comment s'est construite cette traduction bouchère autour de la viande bovine belge et quel en est son contenu. Dans la troisième et dernière partie, le temps du développement de la coopérative et de ses points de vente, nous observons comment la traduction bouchère se fait hybrider par le collectif fermier.

\section{Rencontre entre deux collectifs}

Les quotas laitiers en 1984 et la double spécialisation ensuite, posent aux paysans de Coprosain la question du développement d'une filière spécifique "viande bovine ». Côté consommation, la coopérative se laisse interpeller à l'époque par ses clients qui souhaitent voir leur panier d'achat élargi du poulet fermier au bœuf fermier. Face aux "scandales aux hormones ", la "vente congélateur " connaît un certain succès mais sa légalité est fragile et les perspectives de croissance relatives. Ceci décide la coopérative paysanne à mettre sur pied une structure collective de commercialisation de viande bovine mais cette fois avec pignon sur rue. Reprenant une boucherie, elle engage alors un jeune boucher.

7 Cette insertion d'un intermédiaire, le boucher, va mettre en présence au sein d'un collectif un réseau de production fermière (la coopérative paysanne et son réseau d'éleveurs) et un réseau boucher (une profession, ses compétences, ses normes). Le projet des premiers est d'obtenir, en échange de la qualité fermière de leurs bovins, un prix plus juste supérieur à celui du marché classique.

\section{Controverse : le juste prix ?}

8 La fixation du prix des bovins à abattre, à l'entrée de la boucherie Coprosain, est donc définie selon la logique «du prix équitable». Évitant marchands de vaches et autres chevillards, convaincus de la qualité fermière, c'est sur base d'échanges personnels que la coopérative et les éleveurs ajustent les prix systématiquement supérieurs au prix du marché du bétail de boucherie. Le boucher recruté se préoccupe de son côté du prix de vente au détail de la boucherie coopérative. Il fixe ce prix à partir d'un relevé des prix pratiqués dans les boucheries de la région. Les registres de prix sont disjoints. À la différence de celui du beurre ou du poulet fermier, le prix des bovins repose sur les pratiques de découpe et de transformation que les éleveurs ne maitrisent pas. C'est la logique bouchère. L'absence de lien entre le prix de vente au détail et le prix d'achat des carcasses pose deux problèmes :

1. Un problème d'équivalence de prix : comment fixer le prix d'un kilo de rôti par rapport à celui d'un kilo de carcasse? 
2. Un problème de définition de la qualité de la matière première : comment faire varier le prix d'une matière première dont la qualité varie, sans définition de cette qualité ? Les uns s'appuient sur le prix « fermier », les autres sur le prix « boucher ».

Derrière ces discussions, des qualités différentes sont en jeu. Les équivalences qualité/ prix prolifèrent et menacent la stabilité du collectif, le bilan économique est négatif et après deux ans les comptes de la boucherie fermière plongent dans le rouge. C'est alors que le boucher se décide à intervenir.

Le boucher, qui n'est qu'un jeune salarié au service de quinze éleveurs aguerris, va, malgré son jeune âge et son inexpérience, imposer le silence aux discussions incessantes en définissant un espace de calculabilité. Pour construire cet espace de calculabilité, nous dit Callon, il faut tracer une frontière claire et précise entre, d'une part les relations que l'agent va prendre en compte et qui serviront dans le calcul et, d'autre part, la multitude des relations qu'il ignorera. Pour construire un marché, c'est-à-dire pour transformer quelque chose en un bien marchand, il est nécessaire donc de choisir certains liens auxquels va être rattaché l'objet, mais il est aussi impératif de couper d'autres liens entre cet objet et d'autres objets ou êtres humains, un par un. C'est cette opération de détachement qui permet de cadrer une opération et c'est seulement quand ce détachement est réalisé que le calcul du juste prix peut aboutir (Callon, 1999). Comment le jeune boucher s'y prend-il ? Pour substituer à l'énoncé du juste prix cher aux paysans un calcul noir sur blanc, il va couper ce juste prix de ses lignes d'approvisionnement en objectivant le manque à gagner de la coopérative... des paysans. Ensuite il avancera ses propres pions en reliant le prix d'achat (des carcasses) à des catégories et le prix de vente (au détail) à des ratios morceaux de premier et second choix. Ce n'est plus le jeune boucher inexpérimenté qui parle, mais un calcul « objectif».

Figure 1. Tableau de découpe

\begin{tabular}{|c|c|c|c|c|c|}
\hline \multicolumn{6}{|c|}{$\begin{array}{c}\text { Viande de bouf : carcasse et demi-carcasse } \\
\text { Catégories }\end{array}$} \\
\hline \multirow[t]{2}{*}{$\begin{array}{l}\text { Dénomination des } \\
\text { morceaux }\end{array}$} & & & & & \\
\hline & & 2 & 3 & 4 & 5 \\
\hline 1. Filet pur & $2,1 \%$ & $2,0 \%$ & $1,9 \%$ & $1,8 \%$ & $1,80 \%$ \\
\hline 2. Contre-filet & 2,9 & 2,8 & 2,7 & 2,6 & 2,3 \\
\hline 3. Entrecôte & 4,9 & 4,8 & 4,6 & 3,8 & 3,7 \\
\hline 4. Petite tête & 2,5 & 2,4 & 2,3 & 2,3 & 2,2 \\
\hline 5. Aiguillette & 1 & 0,9 & 0,8 & 0,7 & 0,7 \\
\hline 6. Grosse cuisse & 6 & 5,9 & 5,4 & 5,2 & 4,8 \\
\hline 7. Grosse tête & 3,3 & 3,3 & 3,1 & 3 & 2,8 \\
\hline 8. Balle d'épaule & 2,4 & 2,2 & 2 & 1,8 & 1,7 \\
\hline 9. Pelé qualité I & 2 & 1,8 & 1,6 & 1,5 & 1,4 \\
\hline 10. Faux filet & 1 & 1 & 0,9 & 0,9 & 0,8 \\
\hline 11. Filet d'Anvers & 2,3 & 2 & 2 & 1,8 & 1,5 \\
\hline 12. Plate cuisse & 5,4 & 5 & 4,5 & 4,2 & 3,8 \\
\hline 13. Petit nerf & 1,5 & 1,3 & 1,3 & 1,3 & 1,4 \\
\hline 14. Pelé qualité II & 5,6 & 3,1 & 2,2 & 1,6 & $\overline{1,2}$ \\
\hline 15. Filet américain & 10 & 7,1 & 7 & 6,8 & 6,7 \\
\hline 16. Carbonnade & 7,8 & 8,2 & 8,3 & 8 & 8,6 \\
\hline 17. Bouilli sans os & 3,8 & 4,5 & 4,9 & 4,6 & 2,8 \\
\hline 18. Bouilli avec os & 6,8 & 10 & 9,2 & 9,7 & 11,9 \\
\hline 19. Hâhé & 8 & 7,9 & 10 & 10,8 & 10 \\
\hline $20 . \mathrm{ss}_{\mathrm{s}}$ & 12 & 12,3 & 13,7 & 14,6 & 14,7 \\
\hline 21. Graisse & 8,5 & 11,2 & 11,3 & 12,7 & 14,9 \\
\hline 22. Rognons & 0,2 & 0,3 & 0,3 & 0,3 & 0,3 \\
\hline & 100 & 100 & 100 & 100 & 100 \\
\hline
\end{tabular}


11 Pour aligner les éleveurs, le boucher s'appuie sur un dispositif technico-administratif dont la clef de voûte est un tableau de référence : le tableau de découpe (figure 1). Ce dispositif, contrôlé par l'administration des Affaires économiques, qui luttait à l'époque contre une inflation à deux chiffres, impose aux bouchers un plafond à leur marge brute c'est-à-dire la marge maximale entre prix d'achat des carcasses (par kilo) et prix de vente global au détail (un prix moyen au kilo). Dans les limites de ce premier cadrage, le boucher a néanmoins la possibilité de jouer de l'hétérogénéité des carcasses. Le ministère des Affaires économiques en accord avec la Fédération nationale des bouchers reconnaît en effet 22 types de morceaux différents dont la marge totale est plafonnée mais dont les marges individuelles peuvent varier à l'intérieur de ce cadre de calcul. De plus, la définition des 22 types de morceaux permet aux bouchers et consommateurs belges de coordonner leurs compétences autour de ces références communes. Ainsi l'accord sur le bon " pelé » permet aux premiers la bonne découpe et aux seconds les usages culinaires adaptés. Mais ce qui nous intéresse ici, c'est la hiérarchie que ces 22 types de morceaux, ainsi stabilisés entre bouchers et consommateurs, permettent de définir au niveau des carcasses. En effet, plus il y a de «bons » morceaux c'est-à-dire des morceaux à cuisson rapide, meilleure est la catégorie de carcasse. Ce qui distingue donc les catégories de carcasses c'est la proportion (exprimée en \% de découpe) de beaux morceaux et de morceaux de second choix. Ainsi les proportions de pelé baissent-elles de $30 \%$ en passant de la catégorie 1 à 5 , et celle de graisse, selon la même logique, s'accroissent de $60 \%$.

Revenons à notre jeune boucher : connaissant le prix de vente des différents morceaux qu'il affiche à son comptoir, il peut, selon la catégorie de carcasse et la table de découpe, calculer très rapidement au prorata des pourcentages de morceaux et du poids carcasse le revenu total de ses ventes. Ensuite, soustrayant la marge plafonnée imposée par l'administration, il peut, à reculons, calculer le prix théorique d'achat des carcasses et du bétail sur pied. Le résultat est cinglant: le prix calculé est $15 \%$ inférieur au prix qu'il donne aux paysans de Coprosain. Le manque à gagner est pour la coopérative, dont on comprend pourquoi elle est au bord du dépôt de bilan. Comme les paysans de Coprosain, nous avons aussi du mal à suivre le calcul lié à la découpe et au classement de carcasses. C'est complexe mais implacable. Le calcul du jeune boucher a épuisé les remises en cause permanentes des éleveurs. Séduits, impressionnés, ou virulents opposants, tous sont obligés d'admettre que le boucher a transformé la subjectivité du prix des bêtes, le prix juste, en une définition objective. La controverse est ainsi close, les disputes incessantes épuisées. Ce que les paysans de Coprosain avaient appelé de leurs vœux lors des réunions qui décidèrent de la reprise de la boucherie est enfin réalisé : «S'adjoindre quelqu'un qui connaît le métier, en qui les producteurs ont confiance pour avoir un avis qui soit objectif». Le caractère objectif signifie en fait que les liens entre les représentés, les bovins et les morceaux de viande, et celui qui s'exprime à leur place, le boucher, résistent à toute tentative pour les briser (Latour, 1995). Le contester devient hors prix et reviendrait à réformer les dispositions du ministère des Affaires économiques, et à créer une nouvelle école de boucherie. Mais pour le boucher, l'affaire n'est pas gagnée. S'il est convaincu du bien-fondé de son calcul, il ne peut baisser directement le prix d'achat des bêtes sans risquer de heurter de front la notion de juste prix à la base du projet paysan. 


\section{Aligner les éleveurs}

13 La traduction réussie crée un espace commun, une équivalence, une commensurabilité, elle aligne. L'alignement est parfait lorsque ce que dit le boucher des paysans et de luimême est ce que les paysans disent d'eux-mêmes et du boucher. Ce que dit A de A et de B est ce que dit $\mathrm{B}$ de $\mathrm{B}$ et de $\mathrm{A}$. Les opérations d'alignement aboutissent à des reconfigurations variables du collectif. Ce que nous cherchons à déterminer dans la rencontre entre les traductions fermière et bouchère, c'est l'asymétrie ou l'hybridation que produit la nouvelle traduction. Soit l'alignement est asymétrique, l'alignement est unilatéral: A (les éleveurs) se déplace et s'aligne sur B (le boucher), B ne bouge pas. Le plus faible se détourne et rejoint les intérêts du plus fort et le plus fort déplace et aligne le plus faible sur ses intérêts. Ceci d'autant plus facilement qu'il peut se saisir d'une boîte noire. Soit l'alignement est hybridation, A et B sont déplacés, dans des mesures plus ou moins comparables. Des buts nouveaux sont assignés à l'entreprise qui nécessitent une redéfinition simultanée des identités de A et $\mathrm{B}$. Si les éleveurs ne peuvent baisser leur prix, le boucher peut, en revanche, trier les carcasses et ne donner de bons prix qu'aux bonnes carcasses, la coopérative garantissant après tout un débouché à toutes les carcasses dont le prix moyen reste de $10 \%$ supérieur à celui du marché. Poussés par ce prix incitatif, les éleveurs s'alignent progressivement sur les meilleures catégories de carcasses. Ils glissent progressivement de la colonne 4 vers les colonnes 2 et 1 dans le tableau de découpe. Mais en glissant de la sorte au contact de la table de découpe ils se transforment : cherchant maintenant non plus le meilleur prix mais la meilleure colonne du tableau de découpe, ils recherchent les morceaux de premier choix c'est-à-dire un bétail conforme au souhait du tableau de découpe, un bétail bien conformé.

Dans ce glissement se joue la transformation du métier des paysans. La comparaison dans les cahiers de charges de production de 1987 à 1996, à la rubrique alimentation du bétail signale en effet une rupture. Dans l'énoncé initial, c'est un travail d'éleveur qui se réfère à un système conceptuel «fermier » basé sur le concret de l'activité des éleveurs, plutôt qu'à un calcul. Une coupure nette existe entre ce que l'on maitrise, tout ce que l'exploitation (son propre travail dans les champs) peut apporter aux bêtes (foins, ensilage, paille), et ce qui vient de l'extérieur, les concentrés. Cette coupure est renforcée par la frontière établie entre alimentation et additifs alimentaires. Ces derniers sont définis selon leur origine industrielle, ils s'ajoutent de l'extérieur. L'éleveur raisonne en terme de repas à partir de son univers de production, la ferme, et de ce qu'elle peut donner. Chronologiquement, l'élevage vient en second en fonction des limites de ce que l'exploitation peut apporter. Neuf ans plus tard, l'énoncé bascule de fermier-éleveur à éleveur-engraisseur. Il est alors réorganisé autour d'un objectif abstrait: la ration d'engraissement. L'alimentation n'est plus une succession de différents éléments qui suivent la logique chronologique de distribution du repas mais c'est un tout, qui fait l'objet d'un calcul, le calcul de ration. Une ration qu'il s'agit de composer, de supplémenter et d'équilibrer en fonction de besoins, les besoins de l'engraissement. Les oligo-éléments participent également à cette logique de calcul-ration: ils sont maintenant des compléments qui équilibrent la ration et non plus des additifs à ajouter de l'extérieur. Cet alignement effectué par la traduction du boucher ne se limite pas à l'éleveur, il aligne comme nous allons le voir plus loin l'ensemble du système de production en rendant irréversibles certains choix. 
la traduction fermière initiale sur la traduction bouchère en une traduction engraissement. Ce réalignement est unilatéral : les éleveurs A sont ramenés sur la qualité bouchère. Ils s'alignent sur $B$, le boucher, en devenant engraisseurs, et $B$ ne bouge pas. En se ralliant au jeune boucher, les éleveurs révèlent l'asymétrie de la traduction (Vinck, 1992). Mais quel est le contenu de cette traduction bouchère? Poser la question de son contenu, c'est-à-dire celui de la définition de la qualité bouchère, c'est poser simultanément, la question de sa force, celle qui permet au boucher de s'imposer malgré sa jeunesse.

\section{La traduction bouchère}

16 Comment la découpe belge peut-elle imposer aux éleveurs de Coprosain sa traduction de la qualité ? Quels sont les éléments de la construction de la traduction qui ont amené divers intérêts à s'agréger pour produire finalement, en 1975, un objet et un espace de calculabilité : le tableau de découpe ? C'est en remontant le cours des négociations que nous allons comprendre la «nature » de cette traduction et la force qui lui permet de se généraliser en imposant son objectivité.

\section{Du local au national : la découpe belge}

17 John Law décrit comment pour se rendre maîtres de la route des épices, les marins portugais ont créé au $\mathrm{XvI}^{\mathrm{e}}$ siècle un environnement dans lequel ils ont pu se mouvoir sans menacer l'intégrité de leurs vaisseaux. Il montre ainsi comment l'usage de tables de références et d'un instrument de mesure permet de se situer et donc de naviguer en pleine terra incognita (Law, 1986). La situation des bouchers à la fin des années soixante est semblable à celle des marins portugais. Face à un troupeau belge hétérogène, leur Fédération nationale cherche à atteindre un triple objectif :

- inventer un véhicule qui rende les carcasses mobiles, pour pouvoir les négocier à tout moment ;

- maintenir leur intégrité de façon à les mouvoir sans distorsion ;

- les rendre combinables pour que taureaux et vachettes, Pie Rouge des Flandres et Pie Noire deviennent cumulables et comparables.

La Fédération nationale transforme alors son réseau national de boucheries en laboratoire à l'échelle du royaume. Elle organise en de multiples points sur de multiples carcasses des découpes soigneusement pesées. Le protocole de cette expérimentation précise: «En 1974-1975, des experts du ministère des Affaires économiques, la Fédération nationale et des arbitres neutres ont exécuté un grand nombre de découpes contradictoires, sur base desquelles furent établis des tableaux de découpe, qui ont admis comme point de départ le calcul du prix »'. L'expertise contradictoire est mise sur pied pour garantir " l'objectivité » de la démarche, les intérêts des contribuables et des consommateurs sont en effet en jeu à travers la fixation des prix au détail. Le travail empirique est modélisé selon les deux paramètres que nous connaissons maintenant grâce au jeune boucher de Coprosain : la catégorie de carcasse, et le type de morceau ou dénomination. Les experts obtiennent la mesure de la catégorie de carcasse par l'instrument qui fournit l'équivalence bête sur pied/qualité bouchère des carcasses, c'est-à-dire le prix d'achat à l'abattoir qui réfère à une des 5 catégories. De même ils obtiennent la valeur de la mesure du type de morceau

Économie rurale, 286-287 | Mars-juin 2005 
par l'instrument qui fournit l'équivalence carcasse/type de morceau, c'est-à-dire la découpe. En croisant ces deux paramètres, on obtient un tableau à double entrée catégories/types de morceaux, qui cartographie de façon orthogonale chaque carcasse. Cumulée de découpe en découpe, objectivée par l'expertise contradictoire, la valeur moyenne de ces données sera ensuite validée par un arrêté du ministère des Affaires économiques.

Le tableau de découpe ainsi créé est un mobile immuable et combinable (Latour, 1995). Mobile parce que l'on peut déplacer les carcasses ainsi classées où l'on veut : débattre en leur présence du futur forfait annuel avec l'administration, les stenciler, pour multiplier les feuilles de gestion chères au boucher de Coprosain et les insérer dans tous les manuels de boucherie. Ces mobiles sont immuables parce que, sous cette forme de papiers, ils ne risquent plus l'altération à laquelle chaque déplacement les exposait. Ces mobiles immuables sont combinables parce que les chiffres qui les représentent sont cumulables et permettent d'extraire la catégorie pertinente. D'un seul coup d'œil, les responsables de la Fédération nationale des bouchers contemplent maintenant le troupeau bovin belge, ses prés, ses fermiers et ses races. Rang par rang, catégorie par catégorie, ils peuvent effectuer la revue de ce qui nous apparaissait comme une masse hétéroclite errant sans but. Ils tiennent ainsi d'un côté les 100000 troupeaux du royaume mais aussi de l'autre les 10 millions de mangeurs belges. En effet, c'est selon le double classement du tableau que chaque semaine les mercuriales attribuent un prix aux divers types de bovins. Ce prix circulera dans la presse professionnelle des éleveurs et des bouchers, et alignera ainsi d'une part les habitudes alimentaires et le portefeuille des mangeurs et d'autre part les objectifs de production des éleveurs-engraisseurs rivés sur la hiérarchie de carcasses qu'impose le tableau. Le prix qui circule suffit-il à tenir les carcasses du royaume? Comme le montre la figure 2 (cf. p. 34, Économie rurale, version papier), le prix permet de coordonner verticalement le réseau qu'organise le tableau de découpe. Mais c'est bien la découpe belge, horizontalement dans notre schéma, qui tient les carcasses de bovins (achetées à l'abattoir) au steak et autres carbonades du mangeur belge (vendues à l'étal du boucher). Quelle est donc cette découpe qui coordonne abattoirs et comptoirs de détail?

La découpe belge est une découpe possible. Elle fait des choix sur lesquels le tableau reste muet mais que les manuels de découpe belge révèlent invariablement à travers un vocabulaire technique plus proche du travail de chirurgien que de celui de l'artisan. La découpe y est qualifiée d' "anatomique " (Van der Aa, 1991) et décrite comme une opération de "dissection». Ainsi affirme-t-on que le succès de la race belge, est " redevable aux bouchers qui ont réussi à maitriser la dissection et la mise en valeur de divers muscles issus d'une découpe anatomique soigneusement étudiée... ». Sur quels principes repose cette découpe?

- Le premier principe consiste à suivre scrupuleusement le fil du muscle, à respecter son unicité et ainsi d'éviter de mélanger des muscles aux propriétés variables qui pourraient altérer l'uniformité de ce que les bouchers veulent standardiser. Ce choix chirurgical est bien illustré par la comparaison de la représentation de la découpe anatomique avec celle d'une découpe à l'ancienne ou de la découpe parisienne. Là où aujourd'hui, le boucher belge extrait la masse musculaire en respectant son intégrité, autrefois comme ailleurs, c'est au travers de la masse musculaire que passe le couteau (figure 3). 
Figure 3. Représentations de la découpe "anatomique" et de la découpe à l'ancienne

21

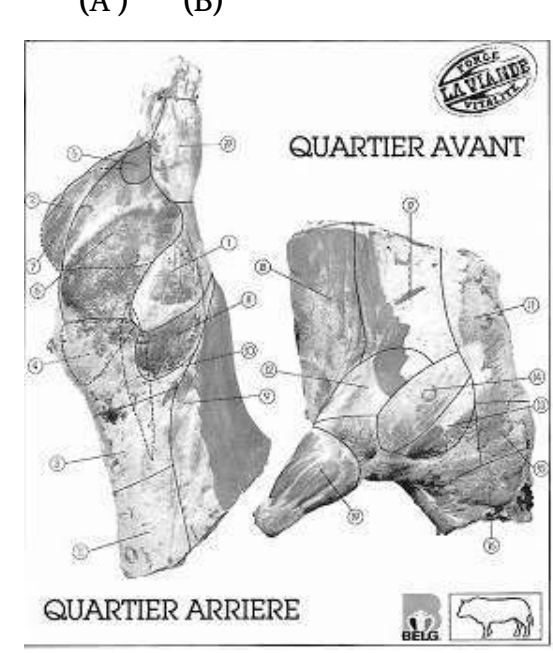



Sources : (A) Découpe anatomique, Van der Aa (1992). (B) Découpe à l'ancienne, Vinck (1948)

Les discussions animées qui ont opposé administration et bouchers lors de l'épreuve contradictoire qui a permis la construction du tableau de découpe, font apparaître le second principe qui guide la découpe anatomique. Aux yeux des inspecteurs, qui défendent consommateurs et contribuables, le parage est sujet à discussion. S'il donne lieu à des réductions de rendement, il influence la marge imposable du boucher. Pour les bouchers en revanche, après avoir dissocié et ainsi respecté le muscle, il s'agit de le purifier ou de le parer, c'est-à-dire de le débarrasser de tout ce qui souille sa pureté : graisse, nerfs, peau et pointes nerveuses C'est ce que certains appelleront le principe de parage à l'excès. Parer permet de présenter une viande d'une couleur et d'une tendreté irréprochable. Cette purification qui soustrait à la viande le gras et le nerf et qui appelle l'uniformité de couleur fait encore aujourd'hui la fierté des bouchers belges. Ce double principe de la découpe anatomique établit un standard de qualité belge : une viande à la fois maigre et tendre. Mais la force du maigre et du tendre est-elle uniquement redevable au travail chirurgical du boucher belge, ou repose-t-elle sur une alliance plus large ? Si le boucher établit une équivalence claire entre sa technique de découpe et la conformation des carcasses, comment, au-delà des carcasses attendues, celle-ci s'étend-elle aux bovins et à leur race?

\section{Inscrire le maigre et le tendre dans une race « hyper viandeuse »}

Depuis le début des années soixante, le petit monde belge de la sélection bovine viandeuse subit d'importantes transformations. On peut y voir une triple rupture qui dessinera les contours d'une performance unique à l'échelle planétaire : la classe $\mathrm{S}^{1}$ ou super cularde. Celle-ci correspondant au sommet de la hiérarchie du tableau de découpe, la catégorie $^{2}$. Le premier point de rupture est le plus radical : la pratique systématique de la césarienne. Avec l'aide d'une cohorte de vétérinaires, cette limite qu'aucune autre race n'a osé franchir est transgressée. Elle ouvre la porte à une réaction en cascade : sélection systématique de reproducteurs possédant le gêne " $m h$ » culard, développement de l'engraissement de culard et en fin de compte, accroissement des rendements bouchers : gain de $10 \%$ sur les rendements carcasses et de 30 à $40 \%$ sur les morceaux de premier 
choix. Le verrou de la naissance par voie naturelle ayant sauté, l'idéal du muscle porté par la découpe anatomique s'accommode alors de l'usage généralisé des anabolisants dans les pratiques d'engraissement ${ }^{3}$ (Leathes et Terry, 1985). «Ben Johnson » des races à viande, la race nationale Blanc-Bleu Belge culard devient ainsi l'archétype d'une trajectoire artificialisante de l'élevage bovin... et c'est cette trajectoire que les éleveurs fermiers de Coprosain sont contraints de suivre pour devenir éleveurs-engraisseurs. Enfin, le modèle de finition est conçu pour livrer des bêtes " prêtes à trancher ». Attendries et mûries sur pied grâce à des techniques très pointues d'engraissement et de finition, les jeunes carcasses de taurillons, parce que maigres et tendres, font alors l'impasse sur la maturation en frigo. Ce dernier point est moins spécifique au Blanc-Bleu Belge. Il est davantage une accélération de la trajectoire d'artificialisation dessinée par les deux ruptures précédentes. Elle produit de jeunes taureaux en stabulation, nourris « ad libitum " à base de concentrés, poussés pour faire des gains de poids intenses, finis idéalement autour de 18-20 mois, appréciés de la grande distribution qui grâce à ces jeunes bêtes trouve régularité des formats, tendreté de la viande et homogénéité de sa couleur.

$\mathrm{Au}$ niveau national, ces ruptures et accélérations vont créer une bifurcation dans l'évolution des races bovines. Issu de la race de moyenne et haute Belgique, le Herd-Book ${ }^{4}$ «Bleu-Blanc Belge » est créé en 1973. Deux ans plus tard, en 1975, année où le tableau de découpe est reconnu par arrêté ministériel, le Herd-Book Blanc Bleu Belge choisit l'orientation cularde hyper viandeux: le Herd-Book du Blanc Bleu belge culard est né. L'alliance entre Blanc Bleu belge culard et Fédération nationale des bouchers est scellée. Cette rencontre n'est pas le fruit du hasard des calendriers. Elle est le produit d'une densification toujours plus intense du maigre et du tendre qui s'inscrit dans la physiologie même de la race : la génétique et le modèle d'engraissement produisent une musculature précoce, une adiposité et une maturité plus tardive. De plus, l'usage des anabolisants a pour effet zootechnique d'augmenter la vitesse de croissance et la proportion de viande maigre par rapport au gras (Maghin Rogister et de Brabander, 1999). Nous comprenons alors mieux comment le tableau de découpe a inscrit jusque dans la physiologie de la race Blanc-Bleu Belge culard la force du maigre et du tendre et pourquoi les paysans de Coprosain, outre la nouvelle école de boucherie, devraient s'atteler à construire une nouvelle race dans les plus brefs délais s'ils avaient voulu refuser l'alignement qu'impose le tableau de découpe. Ainsi réduit au maigre et au tendre, le projet fermier veut-il s'en accommoder dans la durée ou au contraire à son tour intéresser bovins et bouchers à son propre projet pour les hybrider?

\section{L'hybridation fermière}

Pour trancher la question de l'hybridation fermière de la définition de la qualité, nous devons démontrer que la traduction du maigre et du tendre est transformée sous la poussée des différents éléments du collectif Coprosain ou autrement dit montrer comment $\mathrm{B}$ (le maigre et le tendre) va à son tour être dévié par les intentions et projet de $\mathrm{A}$ (le collectif fermier). Montrer comment ce projet traduit à son tour B, c'est montrer à la fois comment, et sur quoi, portent ces transformations.

Le projet fermier de Coprosain n'est pas neutre. Il entretient d'étroits liens avec le mouvement ruraliste et s'est construit en opposition au modèle corporatiste et sectoriel porté par la PAC. Fondé sur l'idée d'autonomie paysanne et de maîtrise du métier, il est proche « du monde n'est pas une marchandise » de José Bové. Mais au-delà de la rhétorique, 
comment assure-t-il la cristallisation de ces valeurs dans une transaction que dominent le maigre et le tendre? Comment la conscience du collectif fermier peut-elle s'exprimer dans le produit alors qu'a priori, dominée par les impératifs économiques du maigre et du tendre, elle ne s'exprime que de façon diffuse et fragmentée ? Ce qui caractérise le projet fermier de Coprosain, ce n'est pas une forme d'organisation ou un produit. C'est au contraire un modèle flexible, inachevé dont les produits phares se transforment : hier un groupement et son poulet fermier à l'étal du marché, aujourd'hui du bœuf vendu à travers une boucherie coopérative et demain une entreprise familiale et ses charcuteries fermières. Ce que certains tentent de saisir à travers un instantané comme une forme d'organisation stable dans ses structures et sa gamme de produits est au contraire une forme d'organisation changeante (Stassart, 2003). Mais comment cette instabilité apparente peut-elle durer ? Pour répondre à cette question, il faut rechercher le modèle, non pas dans la pérennité de ses structures ou de ses produits, mais davantage dans ses modalités d'assemblages, modes de mise en ordre que décrivent des principes d'action (Law, 1994). Ces principes sont des règles d'engagement qui guident l'action et définissent les modalités d'assemblage du collectif. La notion d'engagement est ce qui permet de comprendre aux niveaux individuel comme collectif, l'énergie et la compétence qui sont présentes dans l'action et qui sont ce qui engage la responsabilité (Wynne, 1992). L'engagement postule un ordre de relation qui ne relève ni du calcul stratégique, ni de la rationalité logique mais de l'ordre du lien et de la place de l'individu ou du groupe dans ces liens en terme de responsabilité et d'obligations. Ces règles d'engagement du collectif fermier, nous pouvons les décrire à partir de l'observation des modalités selon lesquelles le maigre et le tendre se transforment ${ }^{5}$.

\section{Non-exclusion des petits et inscription dans l'histoire fermière}

27 La coopérative fermière, contrairement à la boucherie traditionnelle, ferme son réseau. Les transactions classiques entre éleveurs - marchands de vaches - engraisseurs chevillards/abattoir et abattoir/bouchers s'inscrivent dans une logique marchande qui lie et délie selon le succès ou les revers les acteurs. Ceci contraint les intermédiaires à garder leur réseau d'éleveurs-fournisseurs ouvert. Parce qu'ils s'appuient précisément sur l'hétérogénéité de l'offre et la diversité de la demande, les intermédiaires marchands et/ ou chevillards entretiennent une asymétrie d'information dont la contre partie est la possibilité à chaque moment de fermer à certains et d'ouvrir à d'autres leur marché (Stassart, 2003). C'est la logique des « bons et mauvais coups » à réaliser ou à encaisser. La coopérative Coprosain, à l'opposé, produit une double fermeture qui traduit deux règles d'engagement distinctes. La première concerne l'accès au marché. Si Coprosain organise le circuit court passant de cinq opérateurs à deux (éleveurs-engraisseurs et abattoirbouchers), il organise encore davantage le circuit fermé. Contrairement à ses collègues, le jeune boucher, s'il peut trier les carcasses pour en fixer le prix, ne peut en exclure et/ou aller voir ailleurs pour trouver meilleur prix ou meilleure qualité. C'est sur cette obligation d'associer tous les éleveurs que la coopérative fait la différence avec le fonctionnement du marchand ou du chevillard. Elle inclut ainsi tous ses membres dans une dynamique progressive de négociation et d'amélioration que les éleveurs acceptent en échange de l'assurance d'un débouché. Cette fermeture inclusion de tous les éleveurs repose sur la règle qui engage de façon radicale le boucher dans le projet fermier : la non exclusion du petit producteur, $d u$ plus faible (règle 1). Cette règle permet de maintenir un engagement en faveur de l'intérêt commun contre la logique des intérêts les plus 
puissants. Ensuite, et ceci est le second déplacement que la coopérative fermière impose au boucher par le circuit court, si le tableau de découpe a effectivement cartographié le monde de la viande bovine, c'est une tout autre cartographie qu'elle propose à ses consommateurs. Ainsi, dans le «circuit de la viande» et les portes ouvertes que la coopérative organise, c'est l'origine fermière et non le travail boucher qui est pointée comme première responsable de la qualité du produit. Ceci pose la question de l'attribution ou de l'imputation de la qualité. Si par un premier mécanisme, le maigre et le tendre ont su recruter les alliés qui lui ont permis de s'imposer, il existe un second mécanisme par lequel Coprosain, malgré cette traduction du maigre et du tendre, attribue le résultat de ce travail à la coopérative fermière c'est-à-dire à son réseau d'éleveurs. Le bœuf est fermier et non boucher. Il s'inscrit dans l'histoire «fermière ». Mais comment ce travail d'attribution, de circulation de l'histoire fermière est-il assuré ?

Coprosain, comme collectif « circuit court », impliquant éleveurs, bouchers et carcasses, mais aussi fermes et consommateurs, articule deux modalités de circulation ou plutôt de régénération de ces valeurs : la réciprocité et la bienveillance. La réciprocité défiant la fragilité de la loyauté individuelle instaure une loyauté collective: en collaboration étroite avec des groupements de consommateurs (groupements d'achat); des portes ouvertes à la ferme sont régulièrement organisées et agrémentées par des repas débats et des visites à la coopérative. Elles permettent de confronter un collectif de paysans (coopérateurs) à des collectifs de consommateurs diversifiés mais engagés : émanation du syndicalisme ouvrier, du mouvement autogestionnaire ou de l'écologie urbaine. L'extension des réseaux de commercialisation rend cependant cette réciprocité du face à face producteurs/ consommateurs incertaine. En multipliant sa présence dans les fêtes de villages et d'associations locales, la coopérative assure à ses produits une renommée qui repose sur une mise en scène de l'histoire de Coprosain dans un cadre festif : bœuf à la broche, travail d'équipe, assiette fermière. Ce dispositif s'appuyant autant sur les sens et que l'expérience permet en articulant dimensions cognitives et sensorielles, de cristalliser une histoire que le produit ne dit pas, un projet et des valeurs dont témoigne la coopérative en pratiquant ici l'économie de la modération (Karpik, 1989). L'organisation et le prix modeste témoignent en effet d'une forme de rentabilité sociale plutôt qu'économique qui se veut témoin de son projet « d'agriculture sociale ». Ce projet repose sur des valeurs de solidarité entre groupes de consommateurs et petits producteurs, et des notions d'équilibres naturels (élevages liés à la terre) et sociaux (ville - campagne).

Ce travail de circulation repose donc sur une seconde règle d'engagement: la règle $d$ 'inscription dans l'histoire du collectif fermier (règle 2). Elle est bien plus qu'une opération de communication: l'attribution du bœuf (au) fermier et non $(\mathrm{au})$ boucher ré-ouvre un espace engageant le dialogue entre éleveurs paysans et consommateurs. Mais cela suffit-il pour qu'au-delà de l'organisation, $\mathrm{B}$, le maigre et le tendre ainsi déviés par les intentions de A, les paysans éleveurs, se transforme en un nouveau produit?

\section{Exclusion des boîtes noires et ouverture par association}

La traduction du maigre et du tendre est le produit d'une puissante alliance entre boucherie et Blanc-Bleu. Pour les professionnels de l'engraissement, cette alliance s'est nouée autour de l'hyper musculature des bovins et de leur rendement à l'abattage qui fait l'indiscutable supériorité de leur race. Certains profanes avertis ne peuvent pourtant pas 
s'empêcher d'émettre des doutes sur l'avènement de cette race cularde aux formats hors normes. Ainsi une responsable d'un groupe d'achat s'inquiète: "Est-ce qu'il n'y a pas d'hormones? Qu'est-ce que les éleveurs leur donnent aux Blanc-Bleu Belge pour avoir des bêtes comme ça? Je me souviens, je lui demandais à mon cousin ce qu'il leur donnait. Il me prétendait rien du tout, mais je ne pense pas que ce soit ainsi ». La coopérative fermière Coprosain s'est, quant à elle, très rapidement opposée à de telles pratiques. Pour démontrer son engagement, elle met en place un contrat de production strict qui exclura formellement les antibiotiques, les hormones et les facteurs de croissance dès 1987, puis les farines animales en 1989 bien avant la première crise de la vache folle. Elle explorera ainsi différents systèmes de certification de son contrat producteurs-consommateurs à une époque où le principe même d'instaurer le contrôle d'une tierce partie autre que la puissance publique était rejeté. Face aux hormones ou aux antibiotiques, les paysans sont en même temps aussi démunis que les consommateurs : ils savent que ces substances sont condamnées ou condamnables, mais ils ne disposent d'aucun moyen de discuter ou de mesurer le risque. Ainsi, le voudraient-ils, ils n'auraient pas les ressources cognitives qui leur permettraient de discuter de la nature des substances à effet antibiotique, ils les excluent donc selon une troisième règle d'engagement: l'exclusion des boites noires et de ce qui n'est pas négociable (règle 3).

31 La prétention à faire des aliments sains conduit donc à une fermeture la plus complète possible de la filière d'alimentation, fermeture cohérente avec la recherche d'autonomie par rapport aux circuits industriels, car hormones, antibiotiques et farines animales peuvent être assignés à des processus d'industrialisation et de manipulation marchande qui dépossèdent les éleveurs de leur produit. Ce travail d'exclusion des boîtes noires suffit-il? Les frontières sont-elles toujours étanches? Ne faut-il pas admettre dans certains cas, des entrées dans le cycle de production de produits qui complètent l'alimentation produite sur la ferme? Il n'est donc jamais possible d'exclure totalement. Tout à la fois ouvert et fermé, le collectif gère l'incertitude en mettant en négociation toute ouverture de la filière à des intrants exogènes. Ainsi le terme "alimentation fermière » des bovins est une notion frontière qui permet d'entretenir des questions sur "qu'est-ce que produire à la ferme »: produits ou seulement fabriqués sur la ferme, matières premières ou compléments ? Compléments simples ou composés dont on ignore les formules? En maintenant ainsi la tension sur l'aliment fermier d'un cahier de charges à l'autre, le collectif fait tout un travail de négociation sur ce qui peut être inclus. Ce jeu entre aliment fermier et exclusion des hormones autour du bovin sain nous indique selon quelle règle le risque est géré et le sain préservé : l'ouverture par association (règle 4).

C'est par ce principe d'ouverture par association que les éleveurs vont se laisser interpeller par des consommateurs au sujet d'un lien que le maigre et le tendre avaient dissous: le lien entre viande plus foncée et viande plus goûteuse. Posée à l'étal du boucher, débattue aux tables du restaurant que Coprosain crée, cette question sur le retour du goût et la couleur remonte lentement, au cœur de la filière. Mais comment s'affranchir des alliés d'hier : la race hyper musclée et ses modalités d' "engraissement poussé »? Ainsi s'ils y ont songé un moment, les éleveurs-engraisseurs renoncent à unifier leurs formules d'aliments fermiers pour bétail trop liées à leurs firmes de fourniture d'intrants. En revanche, ils finissent par s'accorder pour freiner un engraissement qui, à vingt mois, donne une viande trop claire. S'écartant encore davantage du modèle taurillon, la coopérative développe ensuite ses ventes de génisses ${ }^{6}$ qui, du fait de leur âge et de leurs passages en prairie, produisent une viande plus foncée 
et plus grasse aux qualités gastronomiques appréciées par les amateurs. Ce retour du goût et de la couleur est alors stabilisé dans une grille renouvelée de classification de carcasses qui prend en compte dans la fixation du prix le caractère fermier extensif, en pénalisant financièrement les carcasses jugées trop claires et les bêtes trop rapidement engraissées.

Il y a donc bien transformation de la traduction du maigre et du tendre par la traduction fermière: on freine plutôt que d'accélérer l'engraissement, on retire une partie des taureaux culards pour les remplacer par des vaches d'un ou deux veaux. Mais ces transformations sont hybrides, partielles parce que si $\mathrm{B}$, le maigre et le tendre, a été détourné par les projets fermiers de $\mathrm{A}, \mathrm{A}$ redéfinissant certains points de rupture qui avaient permis à la norme du maigre et du tendre de s'imposer (hormones et systèmes d'engraissement), A c'est-à-dire le collectif fermier ne peut remettre en cause le cadre général formé par la race et la corporation des bouchers. Mais ces transformations hybrides sont $\mathrm{du}$ point de vue du projet fondamentales, parce qu'elles révèlent en quelque sorte sa conscience. Derrière le principe de freiner plutôt que d'accélérer l'engraissement, derrière l'idée de reporter de quelques mois la finition, c'est toute la logique d'artificialisation de l'élevage qui est remise en cause, au-delà du Blanc-Bleu Belge et de ses flirts avec les hormones... Et si nous avions pu dans cet article porter notre regard 15 ans plus tard, nous aurions pu assister à la poursuite du travail, d'ouverture par association, d'inscription dans le projet fermier et d'exclusion des boîtes noires qui amène le collectif à produire et consommer du (véritable) bœuf charolais. B s'alignant un peu plus sur A, travaillant le gras, la carcasse moins conformée et les nouvelles techniques de maturation.

\section{Conclusions}

La construction du marché du bœuf belge est un travail de généralisation qui se stabilise autour d'un objet sociotechnique qui organise et dit en même temps ce qu'est la qualité. Il montre l'importance que jouent certains objets dans la construction d'une filière d'un marché. Nous avons vu comment à travers la controverse, l'objet tableau de découpe a alors créé de la convergence. Le tableau de découpe est un objet intermédiaire (Vinck, 1999) dont la force repose sur trois caractéristiques. Il est d'abord le point d'appui qui permet de transmettre à distance un savoir-faire peu codifié, celui de la découpe anatomique. Il définit et participe à un cadrage général qui aligne le métier des éleveurs, les (in)compétences des mangeurs (du tendre) et les formes préférées de présentation au comptoir (la culture du maigre) pour produire du maigre et du tendre. Enfin il traduit des intentions : une aversion du gras, un rapport à l'animal (la césarienne) qui ne sont pas sans liens avec un projet d'artificialisation de l'élevage. Mais la force du tableau de découpe est aussi sa faiblesse : il est une boîte noire qui devient non négociable. On ne peut pas à chaque fois renégocier le gène culard, la fédération nationale des bouchers ou la classe " $\mathrm{S}$ " des carcasses. Il est donc aveugle et insensible aux changements, il supprime l'espace et le temps. Ainsi en 1996, lorsque tous les regards angoissés se tournent vers l'Angleterre et ses vaches folles, imperturbable, le maigre et le tendre continuent à vanter la qualité diététique de sa viande, comme si le temps s'était arrêté vingt-cinq ans plus tôt, à l'époque où margariniers et producteurs de beurre s'affrontaient sur la valeur diététique de leur gras respectif. C'est alors en référence à ce robuste cadrage, que le caractère alternatif du marché bovin fermier va se construire. 

collectif fermier va fragiliser et recomposer les guidages fonctionnels (Dodier, 1997) proposés par le tableau de découpe pour construire d'autres repères autour desquels il peut se coordonner. C'est cette recomposition que décrit l'hybridation de la troisième section. Ses questionnements sont organiques, diffus mais pas neutres. Ils proviennent des quatre règles d'engagement qui cadrent les possibles et les font circuler dans la filière.

On a donc affaire à une économie de la qualité fondée sur la construction de repères collectifs qui reposent non pas sur une matérialité (la différenciation du produit) mais sur des règles d'engagement. Son efficacité se révèle sur des produits (productions animales) dont les caractéristiques demeurent incertaines. Elles valorisent les économies cognitives des individus à travers un mode de mise en ordre dont les règles d'engagement sont les repères collectifs. Ce que montre le cas de la construction d'un marché alternatif du bœuf fermier belge, c'est la centralité de ces réseaux (d'engagement) dans l'interprétation et la construction sociale du marché. Cette économie de la qualité ne fait pas donc seulement apparaitre le caractère conventionnel des définitions, elle montre également la relation forte entre définition de la qualité et caractéristique du collectif. Le réseau « fermier », du fait de ses règles, est un réseau dont l'objectivation reste incomplète, non repliée sur une boîte noire, ce qui le rend en même temps fragile et moins stable mais cela le rend en même temps sensible d'une part et souple d'autre part.

Un dernier point nous semble essentiel. Il concerne les temps des traductions et leurs conséquences en matière de politique de soutien aux constructions de la qualité. La construction d'un marché alternatif parce que construit en opposition, et donc en référence à un marché qui fait bloc et arrête le temps, procède d'abord du redéploiement de ce temps. C'est ainsi que la traduction fermière hybride progressivement le marché de référence que le transformateur-boucher a imposé. Du temps à rebours qui force la clôture de la controverse au temps que se donne le collectif pour exprimer sa conscience et la traduire dans le produit, apparaissent des rythmes et des échelles radicalement différentes : quinze ans pour le retour du goût et trente pour celui de l'apparition du bœuf... alors que la normalisation du maigre et du tendre gomme un travail de construction similaire qui aura pris de dix à vingt ans lui aussi. Comment intégrer ce jeu du temps dans les politiques de soutien aux constructions de la qualité et quelles en sont les conséquences sur les conditions qui permettent aux filières de se développer sans dissoudre leur spécificité ? Ces questions ouvrent un nouveau champ de recherche où la prise en compte "des temporalités» dans la construction de la qualité deviennent centrales.

\section{BIBLIOGRAPHIE}

Callon M. La protohistoire d'un laboratoire. In M. Callon (Ed.), « La science et ses réseaux », La Découverte, Paris, 1989, p. 66-116.

Économie rurale, 286-287 | Mars-juin 2005 
Callon M. Réseaux technico-économiques et irréversibilités. Figures de l'irréversibilité en économie. $\mathrm{R}$ Boyer (Ed.), Ecole des Hautes Etudes en Sciences Sociales, Paris, 1991, p. 195-230.

Callon M. La sociologie peut-elle enrichir l'analyse économique des externalités ? Essai sur la notion de cadrage-débordement. In D. Foray et J. Mairesse «Innovations et performances - Approches interdisciplinaires », Ecole des Hautes Etudes en Sciences Sociales, Paris, 1999, p. 399-431.

Dodier N. Remarques sur la conscience du collectif dans les réseaux sociotechniques. Sociologie du travail, 1997, n² 2, p. 131-148.

Eymard-Duvernay F. Conventions de qualité et pluralité des formes de coordination. Revue économique, $1989, n^{\circ} 2$, p. 329-359.

Goodman D., Dupuis M. Knowing Food and Growing Food : Beyond the Production-consumption Debate in the Sociology of Agriculture. Sociologia Ruralis, 2002, vol. 42, $\mathrm{n}^{\circ}$ 1, p. 5-22.

Goodman D. Rural Europe Redux ? eflections on Alternative Agrofood Networks and Paradigm Change. Sociologica Ruralis, 2004, vol. 44, n 1, p. 3-16.

Latour B. La science en action. Paris, Gallimard, 1995, 663 p.

Law J. Organizing Modernity. Oxford : B. Blackwel, 1994, 219 p.

Law J. On the Methods of Long Distance Control: Vessels, Navigation and the Portugese Route to India. In Law J. (Ed), "Power, Action and Belief : a new sociology of Knowledge ?", Routledge et Keagan Paul, London, 1986, p. 234-263.

Leathes M., Terry M. HetHormonens chandaal. Roularta, Brussel, 1985, 157 p.

Lemery B. Les agriculteurs dans une fabrique d'une nouvelle agriculture. Sociologie du travail, 2003, vol. $45, n^{\circ} 1$, p. 9-25.

Letablier M-Th., Delfosse C. Genèse d'une convention de qualité, cas des appellations d'origine fromagère. In G. Allaire et R. Boyer, (Eds), « La grande transformation de l'agriculture », Inra Economica, Paris, 1995, p. 93-118.

Lockie S., Kitto S. Beyond the Farm Gate : Production - Consumption Networks and Agri-Food Research. Sociologia Ruralis, 2000, n 40, p. 3-19.

Maghin Rogister P., De Brabander G. Les hormones anabolisantes et les promoteurs de croissance. In A. Clinquart, J. Fabry, M. Casteels, (Eds), « La Viande ? », Bamst Asbl., Liège, 1999, p. 25-55.

Murdoch J., Marsden T., Banks J. Quality, Nature and Embeddedness. Economic Geography, 2000, n - 76 , p. 107-125.

Stassart P. Asymétrie. In " Produits fermiers entre qualification et identité », Peter Lang, coll. Ecopolis, Bruxelles, 2003, p. 31-92.

Stassart P. Produits fermiers entre qualification et identité. Peter Lang, coll. Ecopolis, Bruxelles, 2003, $424 \mathrm{p}$.

Van der Aa W. Manuel du boucher charcutier. Deurne, MIM,1992, $447 \mathrm{p}$.

Vinck V. Manuel Technique pour bouchers et charcutiers. Victor Vinck, Anvers, 1948, 679 p.

Vinck D. Les objets intermédiaires dans les réseaux de coopération scientifique. Revue Française de sociologie, 1999, XL-2, p. 385-414.

Vinck D. Politique de la science et de la technologie du laboratoire aux réseaux. Direction générale Science, Recherche et Développement, Bruxelles, 1992. 
Wathmore S., Thorne L. Nourishing Networks. Altenative geographies of food. Globalising food. David Goodman et M Watts, Routledge, London, 1997, p. 287-304.

Wynne B. Risk and social Learning : Reification to Engagment. In Sheldon Krimsky et Dominic Golding, (Eds), “Social Theory of Risk", London, 1992, p. 44-83.

\section{NOTES}

1. . Le règlement CEE 1208/81 définit un classement «EUROP » des carcasses selon la qualité de leur conformation, basée sur le développement des profils de masses musculaires. Les classes vont de $\mathrm{E}$ (carcasses les plus conformées) à $\mathrm{P}$ (carcasses moins conformées). Le règlement 1026/91 ajoute la classe S des carcasses de "super» culard Blanc-Bleu Belge. Le classement EUROP devient SEUROP et reconnaît ainsi la supériorité des carcasses de Blanc-Bleu Belge culard.

2. . In Van der Aa, Manuel de boucherie, 1992.

3. Au début des années 1990, l'efficacité des contrôles imposera le respect effectif de l'interdiction sans pour autant clore la controverse, qui rebondira plus tard à l'OMC entre l'Union européenne et les États-Unis.

4. . Le Herd-Book est le livre généalogique des races bovines et porcines. Par leur inscription et un système de cotation, il atteste de l'origine des animaux et de leurs performances.

5. . Au contraire du collectif fermier en train se faire, la norme du maigre et du tendre, parce qu'elle est stabilisée, efface derrière son caractère "objectif» les règles d'engagement sur lesquelles repose sa construction. Leurs caractéristiques très générales appartiennent à l'ordre industriel : qualification sur des critères simples, externalisation des effets secondaires (aliment pour bétail), et délégation de la figure du consommateur aux bouchers qui ont présidé à sa construction.

6. . Génisses s'emploie ici pour des vaches d'un ou deux veaux.

\section{RÉSUMÉS}

La construction de la qualité, ici dans le cadre d'une filière fermière de viande bovine, est souvent analysée comme le résultat d'une convention. L'article analyse la confrontation entre un projet paysan avec les normes industrielles de qualité qui dominent le marché belge. Cette confrontation entraîne une hybridation mais surtout elle révèle comment une qualité est liée à une forme de collectif, c'est-à-dire d'engagement des acteurs. L'article montre comment ce sont les principes constitutifs et les règles de fonctionnement du collectif qui président à une définition de la qualité qui vaut moins par sa stabilité et son extension que par sa flexibilité et sa sensibilité aux demandes réciproques des acteurs.

Food quality standards can be analysed as results of a convention process. This paper focuses on a beef farm production and commercialisation chain. It analyses the confrontation between a farmer's project and the industrial norms that are ruling the Belgian beef market. This confrontation leads to a kind of hybridation of quality definition, but the paper shows how quality definition is closely linked with the kind of underlying commitments. Principles and 
criteria of collective organisation are modelling quality standards in such a way that flexibility and sensitivity to mutual demands are more important that stability and generalisation.

INDEX

Code JEL Q56 - Environment and Development; Environment and Trade; Sustainability; Environmental Accounting

Mots-clés : économie de la qualité, filière bovine, hybridation, produit fermier, réseaux agroalimentaire

Keywords : agrifood networks, beef commodity chain, economy of quality, farmer product, hybridation

\section{AUTEUR}

\section{PIERRE STASSART}

Sciences et Gestion de l'environnement, Faculté des Sciences, Université de Liège, Belgique 